\title{
Omental Free Shaped Flap Reinforcement on Anastomosis and Dissected Area (OFFROAD) Following Gastrectomy
}

\author{
WonHo Han, M.D. ${ }^{1}$, KyongLin Park, M.D. ', Deok-Hee Kim, R.N. ${ }^{1}$, Young-Woo Kim, M.D., Ph.D. ${ }^{1,2}$ \\ ${ }^{1}$ Center for Gastric Cancer, Research Institute and Hospital, National Cancer Center, ${ }^{2}$ Department of Cancer Control and Population Health, National Cancer \\ Center Graduate School of Cancer Science and Policy, Goyang, Korea
}

The frequency of anastomotic leakage after gastrectomy is reported to be $0.9 \sim 8 \%$. To reduce deleterious outcomes of anastomotic leakage, we devised the "Omental Free-shaped Flap Reinforcement On Anastomosis and Dissected area” procedure not only to prevent fatal complications following anastomotic leakage but also to promote vascularity of anastomoses and other expected oncological benefits. This video illustrates the surgical procedure following a totally laparoscopic distal gastrectomy. After completion of the anastomosis, the remaining omentum was mobilized upward and divided into two sections. We placed the left section of the omental flap under the anastomosis between the stomach and pancreas. Finally, we grasped and curved the tip of the section to cover the anastomosis from behind, and we placed the right section of the omental flap above the anastomosis. These two sections were approximated with clips to the anterior wall of the stomach. The patient was discharged without complications.

Keywords: Gastrectomy, Gastric cancer, Leakage, Anastomosis, Omental flap

Supplementary video file: This article contains supplementary material (https://doi.org/10.7602/ jmis.2018.21.4.180).
Received June 26, 2018

Revised 1st August 1, 2018

2nd August 21, 2018

Accepted September 4, 2018

Corresponding author

Young-Woo Kim

Department of Cancer Control and

Population Health, National Cancer

Center Graduate School of Cancer

Science and Policy, 323 Ilsan-ro,

Ilsandong-gu, Goyang 10408, Korea

Tel: +82-31-920-1635

Fax: +82-31-920-0069

E-mail: gskim@ncc.re.kr

ORCID:

http://orcid.org/0000-0002-1559-9672

WonHo Han and KyongLin Park contributed equally to this study.

Copyright $\odot 2018$ The Journal of Minimally Invasive Surgery. All rights reserved.
This is an Open Access article distributed under the terms of the Creative Commons Attribution Non-Commercial License (http:// creativecommons.org/licenses/by-nc/4.0/) which permits unrestricted non-commercial use, distribution, and reproduction in any medium, provided the original work is properly cited.

\section{INTRODUCTION}

Anastomotic leakage is a frequent complication in gastric cancer surgery, with a frequency of $0.9 \sim 8 \%$. It is one of the most serious postoperative complications because its mortality rate is known to be as high as $20 \%$.,

We devised an omental flap reinforcement surrounding the anastomosis, considering that it could physically reinforce the anastomosis and, theoretically, mitigate subsequent peritonitis or sepsis even if leakage occurred.

Herein, we report a case of Omental Free-shaped Flap Re- inforcement On Anastomosis and Dissected area (OFFROAD) following reconstruction after a totally laparoscopic distal gastrectomy (TLDG).

\section{PROCEDURE}

The patient was a 55-year-old man diagnosed with gastric cancer in the National Screening Program. Esophagogastroduodenoscopy showed a 3-cm early gastric cancer (EGC) type IIc lesion in the antrum of the stomach along the greater curvature, and the pathological report revealed a poorly dif- 
ferentiated adenocarcinoma. The initial computed tomography did not present any lymph node metastasis, and a TLDG was planned for the patient with the preoperative diagnosis of cT1NOM0 EGC.

Five ports were used. A 12-mm balloon trocar was inserted in the umbilicus area, and the other 12- $\mathrm{mm}$ and 5- $\mathrm{mm}$ trocars were also placed. A D1+ lymphadenectomy was performed on the basis of the Japanese gastric cancer guidelines. Next, an intracorporeal Billroth I anastomosis was performed for reconstruction. We modified the procedure slightly. The delta anastomosis was introduced by inserting the staplers at the end of each stapler line rather than the greater curvature of the posterior wall of the duodenum and the remnant stomach. ${ }^{3}$

After the anastomosis, the omentum was mobilized upward to cover the stomach. For this, we dissected part of the omentum adhered to the peritoneal wall and released it enough to cover the anastomosis area without tension. To create two flaps without compromising the vascular arcade, the omentum was divided through the avascular area in a reversed $\mathrm{T}$ shape into two parts using an energy device. The left part of the omental flap was first placed under the anastomosis, and the tip was retracted out to cover the liver undersurface and anastomosis line from behind. Next, the right part of the flap was placed above the anastomosis, widely covering the whole anastomosis and the edges of two parts of the omentum were approximated. After confirming that there was no tension on any of the organs surrounding the operative area, the approximated flaps were fixed using large metal clips.

\section{RESULTS}

The OFFROAD procedure time was 5 minutes, and there were no procedure-related complications. An esophagogastroduodenoscopy performed on the 2nd postoperative day showed no abnormalities, such as an ulcer or anastomotic leakage. The patient was discharged on the 7th postoperative day with no complications observed during the postoperative period.

\section{DISCUSSION}

We devised and performed the Omental Free-shaped Flap Reinforcement On Anastomosis and Dissected area $\left(\mathrm{OF}^{-}\right.$ FROAD) procedure for gastric cancer surgery to reinforce the anastomosis physically using the omentum, promote healing through enhancing vascular supply, and localize and prevent a serious outbreak of sepsis in the case of anastomotic leakage.

We predict that omental flap coverage of the anastomosis promotes the micro-vascularity of anastomosis site and helps prevent ischemia that may cause anastomotic leakage. ${ }^{4}$ In ad- dition, covering the dissected area with the omentum will promote healing by preventing abscess formation because of a protective effect of the omentum, which will deliver fluids or particles to the macrophages and preventing formation of dead space around the dissected area., ${ }^{5,6}$

In addition, the procedure may also reduce difficulty in future gastric surgeries caused by adhesion between the remnant stomach and adjacent organs, such as the pancreas and liver. ${ }^{5}$ Additionally, repositioning of the omentum could likely increase the vascularity around the anastomosis and dissected area and may be beneficial for adjuvant chemotherapy.

Another theoretical oncological benefit of the procedure is the possible reduction of inflammatory cytokine production, which may influence cancer treatment. Several experimental studies have suggested that the omentum may reduce inflammatory cytokines including T-cell subsets (CD4, CD8, and $\gamma \delta \mathrm{T}$ cells) and proinflammatory cytokines such as IL-6 and IL-12 and enhance anti-inflammatory activity by activation of basic fibroblast (bFGF) and transforming growth factorB1 (TGF- $\beta 1$ ) to accelerate the healing mechanism. ${ }^{7,8}$ Cancer cells are known to respond to cytokines, which could promote growth, attenuate apoptosis and facilitate invasion and metastasis.

One concern during this procedure was the possibility of omental necrosis after partial omentectomy. A rare report of omental infarction following laparoscopic gastrectomy is published. ${ }^{9}$ Given this observation, in this study, we attempted to preserve the feeding vessels of the omentum and dissected the omentum along the avascular plane. It should be careful not to sacrifice the omental branch from both sides when performing partial omentectomy. When dividing the flap into two parts, it should be cut at the center of the branch where the two blood vessels meet and be sure to ligation with a clip to prevent bleeding.

In conclusion, we performed a novel OFFROAD procedure that is simple and could have considerable potential benefits after gastrectomy. In the future, technical refinement and well-designed clinical studies along with biological measures should be performed to demonstrate the procedure's efficacy and safety.

\section{ACKNOWLEDGMENTS}

This work was supported by the National Cancer Center grant NCC-1710120.

\section{REFERENCES}

1) Sierzega M, Kolodziejczyk P, Kulig J, Polish Gastric Cancer Study Group. Impact of anastomotic leakage on long-term survival after 
total gastrectomy for carcinoma of the stomach. Br J Surg 2010; 97:1035-1042.

2) $\mathrm{Hu}$ Y, Huang C, Sun Y, et al. Morbidity and Mortality of Laparoscopic Versus Open D2 Distal Gastrectomy for Advanced Gastric Cancer: A Randomized Controlled Trial. J Clin Oncol 2016;34: 1350-1357.

3) Kanaya $\mathrm{S}$, Gomi $\mathrm{T}$, Momoi H, et al. Delta-shaped anastomosis in totally laparoscopic Billroth I gastrectomy: new technique of intraabdominal gastroduodenostomy. J Am Coll Surg 2002;195:284287.

4) Kingham TP, Pachter HL. Colonic anastomotic leak: risk factors, diagnosis, and treatment. J Am Coll Surg 2009;208:269-278.

5) Wiggins T, Markar SR, Arya S, Hanna GB. Anastomotic reinforcement with omentoplasty following gastrointestinal anastomo- sis: A systematic review and meta-analysis. Surg Oncol 2015;24: 181-186.

6) Agnifili A, Schietroma M, Carloni A, et al. The value of omentoplasty in protecting colorectal anastomosis from leakage. A prospective randomized study in 126 patients. Hepatogastroenterology 2004:51:1694-1697.

7) Matoba Y, Katayama H, Ohami H. Evaluation of omental implantation for perforated gastric ulcer therapy: findings in a rat model. J Gastroenterol 1996;31:777-784.

8) Shah S, Lowery E, Braun RK, et al. Cellular basis of tissue regeneration by omentum. PLoS One 2012;7:e38368.

9) Kim MC, Jung GJ, Oh JY. Omental Infarction Following Laparoscopy-assisted Gastrectomy (LAG) for Gastric Cancer. J Korean Gastric Cancer Assoc 2010;10:13-18. 